\title{
$C$-band disk-loaded-type accelerating structure for a high acceleration gradient and high-repetition-rate operation
}

\author{
T. Sakurai, ${ }^{1,2,}$ H. Ego, ${ }^{2}$ T. Inagaki, ${ }^{1}$ T. Asaka, ${ }^{1,2}$ D. Suzuki, ${ }^{3}$ S. Miura, ${ }^{3}$ and Y. Otake ${ }^{1}$ \\ ${ }^{1}$ RIKEN SPring-8 Center, Kouto 1-1-1, Sayo, Hyogo 679-5148, Japan \\ ${ }^{2}$ Japan Synchrotron Radiation Research Institute (JASRI), Kouto, 1-1-1, Sayo, Hyogo 679-5198, Japan \\ ${ }^{3}$ Mitsubishi Heavy Industries Mechatronics Systems Ltd., Komatsu-dori 5-1-16, \\ Hyogo-ku, Kobe, Hyogo 652-0868, Japan
}

(Received 13 January 2016; revised manuscript received 8 August 2016; published 19 April 2017)

\begin{abstract}
A high-acceleration-gradient linear accelerator (LINAC) for an x-ray free electron laser (XFEL) offers the advantages of a short accelerator length and low construction costs. In addition, the high pulse repetition rate of the LINAC, which can drive multiple $\mathrm{x}$-ray beam lines, provides additional user opportunities for experiments involving XFEL. A $C$-band disk-loaded-type accelerating structure was developed to achieve a high acceleration gradient of $>50 \mathrm{MV} / \mathrm{m}$ and an rf-pulse repetition rate of $120 \mathrm{pps}$, which is twice as high as that of the XFEL facility, SACLA (60 pps). The structure has a quasiconstant gradient and a traveling wave type with an accelerating mode of TM01 $-2 \pi / 3$. To reduce the surface electric fields, we employed a cross section with an ellipsoidal curvature around an iris aperture. The accelerating structure was manufactured for SACLA. High-power rf conditioning was conducted to investigate its performance. Owing to the conditioning, the acceleration gradient reached a value of more than $50.1 \mathrm{MV} / \mathrm{m}$. The structure was operated without any serious issues at a repetition rate of $120 \mathrm{pps}$. The accelerating structures were installed in the dedicated accelerator for EUV-FEL at SACLA beam line-1. Finally, we obtained accelerated electron beams with the structures operated at an acceleration gradient of $41.4 \mathrm{MV} / \mathrm{m}$.
\end{abstract}

DOI: 10.1103/PhysRevAccelBeams.20.042003

\section{INTRODUCTION}

An x-ray free electron laser (XFEL), which can provide light with high peak brightness, femtosecond-region short pulse, and high spatial coherence, outperforms existing synchrotron radiation sources. The linac coherent light source (LCLS) and SPring-8 Angstrom compact free electron laser (SACLA) are operated to make an x-ray laser available to experimental users [1,2]. Following the establishment of LCLS and SACLA, many XFEL facilities have been constructed and commissioned around the world [3-5].

There are two important considerations, when constructing an XFEL facility. First, we should obtain the target electron energy with the acceleration length being as short as possible. This reduces the construction costs of the accelerator and its building. Therefore, it is necessary to use high-gradient accelerating structures. However, a high acceleration gradient is usually accompanied by an increase in surface electric fields in the structure. This phenomenon might increase the probability of electric discharges. Furthermore, it increases the dark current, which occurs

\footnotetext{
*t-sakura@spring8.or.jp

Published by the American Physical Society under the terms of the Creative Commons Attribution 3.0 License. Further distribution of this work must maintain attribution to the author $(s)$ and the published article's title, journal citation, and DOI.
}

from the inner surface of the structure. The dark current causes demagnetization of the permanent magnets used in the undulator [6]. Hence, it is imperative to reduce the surface electric fields.

The acceleration gradient, $E_{\text {acc }}$, per unit length of a constant-gradient (CG) traveling-wave-type (TW-type) rf structure is calculated by

$$
E_{\mathrm{acc}}=\sqrt{\frac{P_{\mathrm{in}} \cdot R_{\mathrm{sh}} \cdot\left(1-e^{-2 \tau}\right)}{L}}
$$

where $P_{\text {in }}$ is the input rf power, $R_{\mathrm{sh}}$ is the shunt impedance per unit length, $\tau$ is the attenuation constant, and $L$ is the length of the structure [7]. Further, $R_{\mathrm{sh}}$ varies as a function of $f^{0.5}$, where $f$ is the operation frequency of the structure. If the inner shape of the accelerating structure and the accelerating mode are fixed, and $P_{\text {in }}, \tau$ and $L$ are constant parameters, then $E_{\text {acc }}$ is proportional to $f^{0.25}$. The use of high frequencies reduces the filling time of the accelerating structure in proportion to $f^{-1.5}$; the stored energy is proportional to $f^{-2}$ of the accelerating structure. To reduce the accelerator length, an accelerating structure with a high acceleration gradient field should be operated at as high a frequency as possible owing to the above-mentioned frequency-proportional relation.

Next we should consider sharing the beam time with many experimental users. This is because an XFEL facility is a light source that uses a linear accelerator (LINAC) that 
can generate a high-quality electron beam. However, unlike a ring photon source, it is not easy for a LINAC-based light source to have many beam lines. An electron beam distribution technique from the LINAC to multiple beam lines is crucial to increase opportunities for user experiments. Multibeam-line operation using a fast switching magnet is under development at several XFEL facilities [3-5,8,9]. However, the pulse rate of the $\mathrm{x}$-ray laser per beam line inevitably decreases in the case of multi-beam-line operation. In SACLA, five beam lines can be constructed in future expansions. In this case, the x-ray laser repetition rate per beam line decreases to one-fifth of the present $60 \mathrm{pps}$ accelerator repetition. To maintain a sufficient repetition rate for each beam line, the accelerator should be operated with a higher pulse repetition rate. In this case, an accelerating structure with a higher acceleration gradient and a higher pulse repetition rate contributes significantly toward increasing opportunities for user experiments while reducing the construction costs of the LINAC. To realize a higher acceleration gradient and a higher rf-pulse repetition rate, a $C$-band accelerator is one of the most promising solutions because of the already established technique.

A $C$-band accelerating structure was initially developed for a linear collider project at KEK [10]. The structure was first used on a large scale at SACLA. Since 2011, 128 columns of the structure have been operated in the acceleration gradient range of $35-38 \mathrm{MV} / \mathrm{m}$ at a maximum repetition rate of $60 \mathrm{pps}$ [11]. In the KEKB project, a $C$-band disk-loaded-type accelerating structure with twice the energy gain of the existing S-band accelerating structure was designed, and it was operated at an acceleration gradient of $45 \mathrm{MV} / \mathrm{m}$ in the KEK injector linac [12]. In recent years, many $C$-band accelerating structures have been developed at several accelerator facilities around the world [13-15]. However, even though the rf breakdown rate of a highgradient accelerating structure, which is proportional to the increase in its surface electric and magnetic fields, generally restricts its operation condition, the high-gradient behaviors of $C$-band accelerating structures, as shown by the experimental results obtained at some institutes [16-18], have not been investigated systematically.

For the above-mentioned reasons as well as to clarify the high-gradient characteristics of the accelerating structure, we have developed a new $C$-band accelerating structure that yields a high acceleration gradient $(>50 \mathrm{MV} / \mathrm{m}$ ), which is higher than that of practically operating linacs. Furthermore, we aim to realize operation with a repetition rate of $120 \mathrm{pps}$, which is twice as high as that of SACLA and the same as that of LCLS. Toward this end as mentioned above, we have developed a new accelerating structure with reduced surface electric fields and lower heat loads at higher repetition rates. This paper describes the design of the new $C$-band accelerating structure as well as the practical performance obtained in our experiments.

\section{DESIGN OF THE ACCELERATING STRUCTURE}

With regard to the design boundary of the $C$-band accelerating structure, the total length, filling time, and attenuation constant of the accelerating structure are key parameters, which should be the same as those of the existing choke-mode-type accelerating structure used in SACLA. This is because the existing accelerating structure of SACLA can be easily replaced with a new one, if any problem occurs in the future. The new $C$-band accelerating structure was designed on basis of the following criteria.

1. To obtain a high acceleration gradient of the accelerating structure using the present $C$-band rf sources, we choose an rf design parameter with high shunt impedance. Considering the above-mentioned boundary condition, we aim to obtain a shunt impedance of $>60 \mathrm{M} \Omega / \mathrm{m}$ using a copper rf cavity.

2. The increase in surface electric fields on a cell wall in the structure leads to an increase in the breakdown rate and dark current in the accelerating structure. The axial acceleration gradient is increased by $20 \%$ owing to the increase in the shunt impedance compared with the SACLA structure, while the surface electric field should be the same owing to the special cavity design.

3. The accelerating structure high-gradient behaviors governed by an rf-pulse heating $[17,18]$ should be confirmed.

4. The heat load of the accelerating structure at 120-pps operation is twice as high as that of the existing $60 \mathrm{pps}$ operation at SACLA. The cooling capacity of the water channels of the structure must be able to deal with this heat load.

To satisfy the above-mentioned criteria, we designed a $C$-band accelerating structure having the cross-sectional and outer views shown in Fig. 1. Table I lists the designed and measured parameters of the $C$-band accelerating structure. The material of the accelerating structure was copper. The designed structure is a disk-loaded and quasi-constantgradient (CG) type. The longitudinal phase advance per unit cell of the structure is $2 \pi / 3$ radian in the TM01 transverse mode. The shunt impedance of the accelerating structure is maximized in the longitudinal $2 \pi / 3$ mode [7]. Because the geometric cell shape of this designed CG structure is simple compared to other wakefield damping structures, such as the waveguide-attached damping structure [19] and the choke-mode structure [10], it is expected to reduce the production costs. Furthermore, it is possible to perform precise phase adjustments by the dimpling method on the cell walls after vacuum brazing [20].

Various methods have been proposed for reducing the surface electric fields [21]. To reduce the surface electric fields on the cell walls in the structure, we introduced a cross section with an ellipsoidal curvature around an iris [17]. The maximum surface electric field, which depends on the axis ratio of the major $(B)$ to the minor (A) semiaxis of the ellipse iris, was estimated. Figure 2 


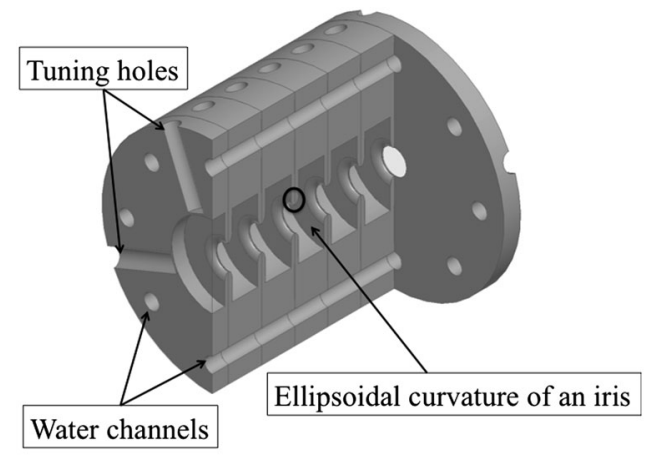

(a)

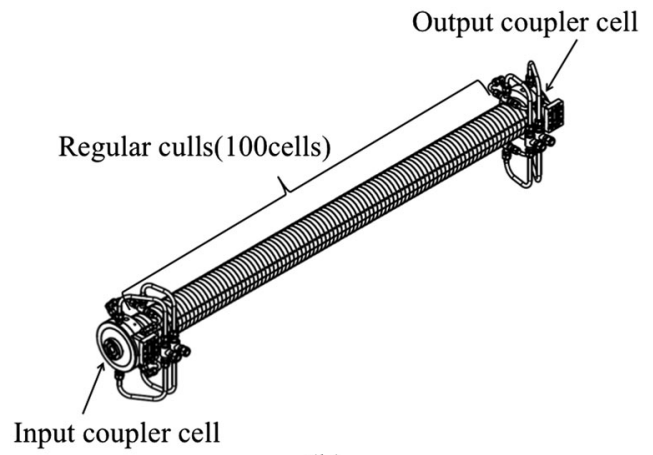

(b)

FIG. 1. (a) Cross-sectional view of the $C$-band disk-loaded-type accelerating structure. (b) Outer view of the new $C$-band accelerating structure.

shows a cross-sectional view of the ellipsoidal iris shape and the ratio $\left(E_{p} / E_{\text {acc }}\right)$ of the maximum surface electric field, $E_{p}$, to the average acceleration field, $E_{\text {acc }}$, as a function of the ratio of $B / A$. The solid line with solid circle dots and the dashed line with open triangles in Fig. 2 denote $E_{p} / E_{\text {acc }} \mathrm{s}$ in the cases of the uppermost $(n=1)$ and the lowermost $(\mathrm{n}=100)$ cells of the accelerating structure, respectively. At $B / A=2.0$, the $E_{p} / E_{\text {acc }}$ values of the uppermost and lowermost cells are 2.81 and 2.57 , respectively. These values are lower than those of the circular curvature case $\left(B / A=1.0 ; E_{p} / E_{\mathrm{acc}} \mathrm{S}\right.$ of the uppermost and the lowermost cells are 3.33 and 2.77 ). These results nearly satisfy the above-mentioned target value. Therefore, we chose $B / A=2.0$. According to these cavity designs, the average shunt impedance of the new structure becomes $66 \mathrm{M} \Omega / \mathrm{m}$.

Furthermore, the possibility of breakdown and surface damage due to an rf-pulse heating was considered. The increase in temperature of the inner surface owing to the pulse heating is calculated as follows [17,18];

$$
\Delta T=\frac{\left|H_{t}\right|^{2}}{\sigma \delta} \sqrt{\frac{t}{\pi \rho c_{\varepsilon} k}}
$$

where $H_{t}$ is the surface magnetic field, $t$ is the length of the rf pulse, $\sigma$ is the electric conductivity, $\delta$ is the skin depth, $\rho$ is the density of the accelerating structure material, $C_{\varepsilon}$ is the specific heat capacity of the material, and $k$ is the thermal conductivity of the material [18,22]. When the acceleration gradient and the length of the rf pulse are $50 \mathrm{MV} / \mathrm{m}$ and $0.3 \mu \mathrm{s}$, the surface magnetic fields at the axis ratios of $B / A=1.0$ and 2.0 in the ellipsoidal iris are 0.11 and $0.10 \mathrm{MA} / \mathrm{m}$, respectively. From Eq. (2), the increase in temperature, $\Delta T$, is 6.2 and $4.7 \mathrm{~K}$, respectively. These values are significantly lower than the breakdown limit described in $[18,22]$, and we assume that such rf-pulse heating does not cause any breakdown or surface damage.

TABLE I. Design parameters and measured values of the $C$-band disk-loaded-type accelerating structure.

\begin{tabular}{|c|c|c|c|}
\hline & & Design parameters & Measurements \\
\hline Structure type & & Quasiconstant gradient, traveling-wave-type & \\
\hline Coupler type & & J-type dual feed & \\
\hline Resonant mode & & TM01 $-2 \pi / 3$ & \\
\hline Number of cells & $N$ & $\begin{array}{l}100 \text { regular cells } \\
+2 \text { coupler }\end{array}$ & \\
\hline Iris diameter $[\mathrm{mm}]$ & $2 a$ & $\begin{array}{c}15.938 \\
\sim 12.107\end{array}$ & \\
\hline Cell diameter $[\mathrm{mm}]$ & $2 b$ & $\begin{array}{c}43.196 \\
\sim 41.869\end{array}$ & \\
\hline Disk spacing $[\mathrm{mm}]$ & $d$ & 17.495 & \\
\hline Disk thickness [mm] & $t$ & 4 & \\
\hline Cross-sectional shape of iris & & Ellipsoidal curvature & \\
\hline Effective length of acceleration [m] & $L$ & 1.767 & \\
\hline Operation frequency [MHz] & $f$ & 5712 & 5712 \\
\hline Average unloaded quality factor & $Q_{0}$ & 9300 & 8900 \\
\hline Average group velocity & $\tilde{v_{g}} / c$ & 0.023 & 0.022 \\
\hline Filling time $[\mathrm{ns}]$ & $t_{F}$ & 290 & 271 \\
\hline Attenuation constant & $\tau$ & 0.59 & 0.54 \\
\hline Average shunt impedance $[\mathrm{M} \Omega / \mathrm{m}]$ & $R_{\mathrm{sh}}$ & 66 & 64 \\
\hline
\end{tabular}




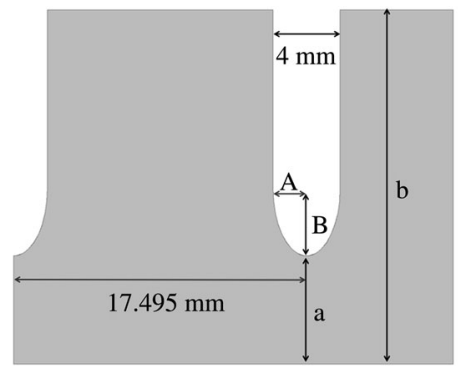

(a)

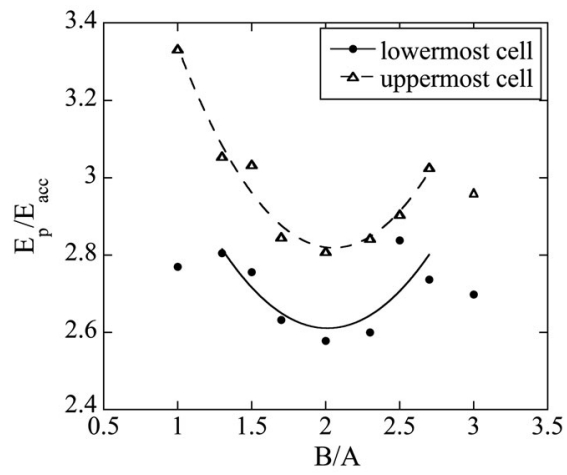

(b)

FIG. 2. (a) Cross-sectional view of the ellipsoidal curved iris. (b) Simulation result of the maximum surface electrical field over $E_{p} / E_{\text {acc }}$ depending on the ratio of $B / A$. The circle and triangle markers show the ratios at the uppermost $(\mathrm{n}=1)$ and lowermost ( $n=100$ ) cell cases, respectively. The solid curve is the quadratic regression curve excluding the points of $\mathrm{B} / \mathrm{A}=1.0,3.0$, and the dashed curve is the quadratic regression curve excluding the point of $\mathrm{B} / \mathrm{A}=3.0$.

We designed the resonant frequency of the cavity cell to be $5712 \mathrm{MHz}$ at a cavity temperature of $30^{\circ} \mathrm{C}$. In SACLA, the allowed $\mathrm{rf}$ phase jitter of the $C$-band main accelerator is less than $\pm 0.5^{\circ}$ [23]. An increase in the heat load of the accelerating structure causes thermal expansion of the cell and shifts the cell's resonant frequency. Consequently, the average temperature and frequency stability of the accelerating structure should have values within $\pm 0.11 \mathrm{~K}$ and $\pm 5.43 \mathrm{kHz}$, respectively, which are determined by the rf phase tolerance of $\pm 0.5^{\circ}$. To realize values within $\pm 0.11 \mathrm{~K}$, we carefully designed a water-cooling method for the structure to effectively transfer the heat and to mitigate both the thermal expansion and the frequency shift of the cell. To estimate the frequency shift, the temperature distribution of the accelerating cell and the thermal and dimensional expansion were simulated using ANSYS [24]. Our simulated thermal design predicts the average heat load of the accelerating structure up to $3.8 \mathrm{~kW}$ at $120 \mathrm{pps}$ operation. This value is equivalent to the heat loss when an input rf power of $80 \mathrm{MW}$ is fed into the accelerating structure. We placed eight water channels having an inner diameter of $8 \mathrm{~mm}$. The water flow rate per channel required by the design is $2.5 \mathrm{~L} / \mathrm{min}$ for realizing the abovementioned power dissipation. The counterflow method is

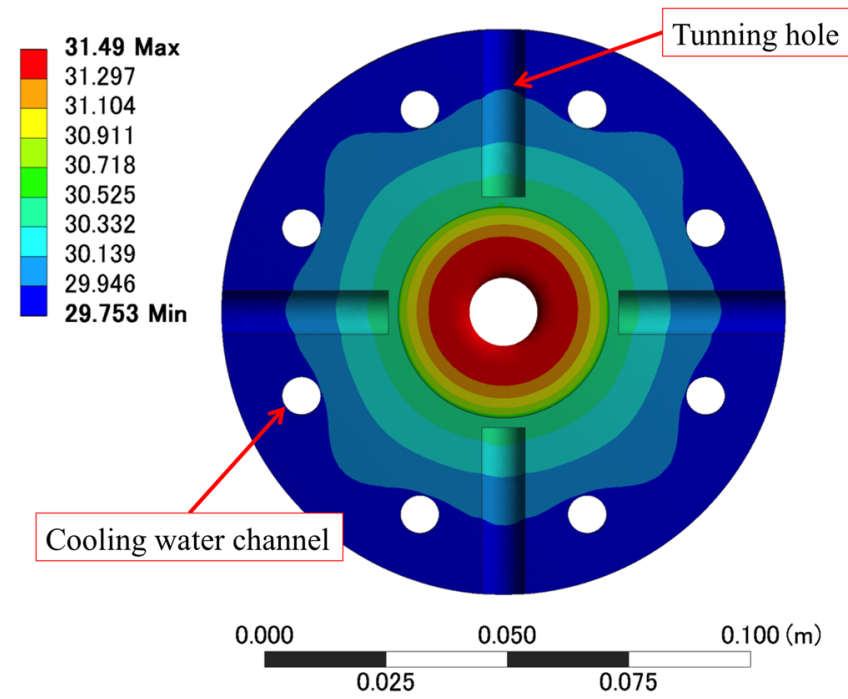

FIG. 3. Estimated temperature distribution of one accelerating cell with a cooling-water temperature of $27.6^{\circ} \mathrm{C}$ at $120 \mathrm{pps}$ operation. The $\mathrm{rf}$ power to be inputted to the accelerating structure is $80 \mathrm{MW}$.

adopted to minimize the temperature gradient along the accelerating structure.

Figure 3 shows the simulated temperature distribution of the accelerating cell at an average structure body temperature of $30^{\circ} \mathrm{C}$ as the designed operating point, with operation repetition rate of 120 pps and a resonant frequency of $5712 \mathrm{MHz}$. In this case, the channel water flow rate is $2.5 \mathrm{~L} / \mathrm{min}$ and the input rf power is $80 \mathrm{MW}$, corresponding to a heat load of $3.8 \mathrm{~kW}$ on the structure. The temperature of the cooling water at an inlet is $27.6^{\circ} \mathrm{C}$ so that the temperature can be maintained at $30^{\circ} \mathrm{C}$. Thus the maximum temperature on the cell surface is $31.5^{\circ} \mathrm{C}$. The diameter of the cell $(2 b)$ increases by around $0.15 \mu \mathrm{m}$, which corresponds to a frequency of $20 \mathrm{kHz}$ lower shifted from $5712 \mathrm{MHz}$. Furthermore, the required temperature stability of the structure is $\pm 0.11 \mathrm{~K}$ as mentioned previously. To maintain the cavity resonant frequency of $5712 \mathrm{MHz}$ at $30^{\circ} \mathrm{C}$ within $\pm 0.11 \mathrm{~K}$ for any rf-pulse repetition rate, we employ a precise temperature regulation system using the proportional integral differential feedback control method, which controls the electric power fed into a heater having a capacity of $7.7 \mathrm{~kW}$ [25]. This system also compensates the lower frequency shift by maintaining the temperature at $30^{\circ} \mathrm{C}$.

\section{NINE-CELL TEST CAVITY}

\section{A. Fabrication}

We fabricated nine-cell test cavities to check the designed rf property of the accelerating structure. The test cavities were checked on the basis of the following points: (i) Check the fabrication method and the surface roughness of the ellipsoidal curvature on the basis of the 


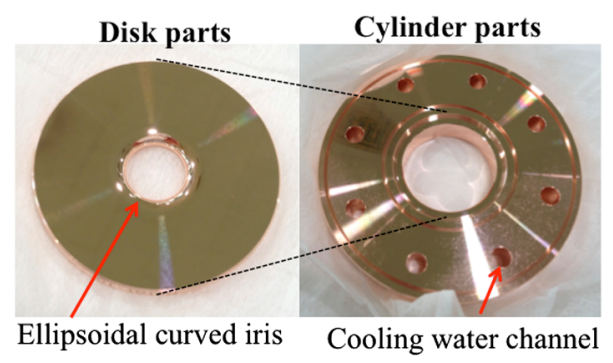

FIG. 4. Disk (left) and the cylinder (right) of the accelerating cell.

cross-sectional shape of the iris. (ii) Check the rf properties of the accelerating structure, such as normalized group velocity, quality factor, and shunt impedance.Three test cavities were machined with different dimensions; their iris diameters (2a) are $15.938 \mathrm{~mm}$ (type 1), $14.003 \mathrm{~mm}$ (type 2), and $12.106 \mathrm{~mm}$ (type 3). These dimensions correspond to the uppermost, midmost, and lowermost iris parts of the accelerating structure, respectively. Figure 4 shows photographs of the disk and cylinder parts that constitute the accelerating structure. The disk surfaces as well as the insides of the cylinder and the ellipsoidal shape of the iris were provided with a mirrorlike finish using an ultraprecision lathe in order to mitigate any concentration of the electric field. After machining, the surface roughness was measured using a three-dimensional shape measurement instrument and a surface roughness meter. The actual ellipsoidal shape of the iris considerably overlapped with the ideal ellipsoidal shape within $10 \mu \mathrm{m}$. The surface roughness of the disk and cylinder parts was around $0.06 \mu \mathrm{m}$, while that of the iris with the ellipsoidal shape was around $0.11 \mu \mathrm{m}$. Even though the ellipsoidal shape is not ideal the maximum dimensional difference from the ideal shape is $4.7 \mu \mathrm{m}$, which is significantly small for our purpose. We cannot show the actual graph data here, because the data include the manufacturing know-how of MHI.

\section{B. Low-power rf test of the nine-cell test cavities}

We checked whether the designed rf parameters of the $C$-band accelerating structure were realized using the test cavities, as follows. This check was also conducted to show the reliability of our accelerating structure design and whether a mass production process of the structure was possible from the trial manufacture stage. The resonant frequency and the quality factor of the TM $01-2 \pi / 3$ mode were measured using the test cavities. The quality factor of the test cavities was estimated from the loaded quality factor, and the coupling coefficient of the accelerating mode was measured by the network analyzer. Figure 5 shows the dispersion curve of the three types of test cavities. The numerically fitted curves in Fig. 5 were obtained using the following formula:

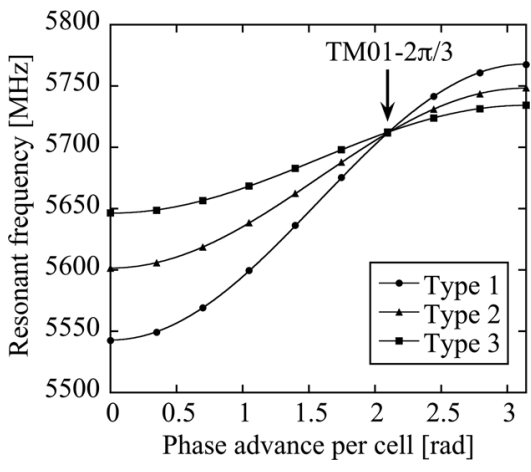

FIG. 5. Dispersion curves of the test cavities.

$$
f=m_{1}-m_{2} \cos (\theta)
$$

where $f$ is the resonant frequency, $m_{1}, m_{2}$ are the proportionality factors related to the group velocity $v_{g}$, and $\theta$ is the phase advance per cell. The term $m_{2}$ denotes the differential coefficient of $v_{g}$. The $v_{g}$ values of the individual cavities are obtained from the dispersion curves using the following formulas:

$$
\frac{v_{g}}{c}=\frac{2 \pi d}{c} \frac{d f}{d \theta}=\frac{2 \pi d m_{2} \sin (\theta)}{c},
$$

where $d$ is the cell spacing and $c$ is the velocity of light [7]. Using the process described above, we obtained the $v_{g}$ values of the test cavities. Table II lists the result of lowlevel rf measurements to characterize the rf performance of the test cavities. The $v_{g}$ values of the test cavities are nearly consistent with our designed values, showing errors within $0.5 \mathrm{MHz}$. The quality factors are $82 \%-90 \%$ of the ideal cavity values. The discrepancies in the values are explained by the electrical contact losses and wall losses of the end plates of the test cavity. The measured $v_{g}$ values are also consistent with the design values.

To estimate the shunt impedance of the test cavities, we measured the electric field distribution along the test cavities using the bead-pull perturbation method. The perturbing object was a sphere made of aluminum, having a diameter of $2 \mathrm{~mm}$. Figure 6 shows the frequency change

TABLE II. Measured results of the nine-cell test cavities. The designed values are stated in parentheses.

\begin{tabular}{lccc}
\hline \hline & Type 1 & Type 2 & Type 3 \\
\hline $2 a[\mathrm{~mm}]\left(30^{\circ} \mathrm{C}\right.$ vacuum) & 15.938 & 14.003 & 12.106 \\
$2 b[\mathrm{~mm}]\left(30^{\circ} \mathrm{C}\right.$ vacuum) & 43.157 & 42.451 & 41.834 \\
Resonant frequency $[\mathrm{MHz}]$ & 5712.145 & 5712.084 & 5711.974 \\
Quality factor & 7400 & 8055 & 7940 \\
& $(8970)$ & $(8910)$ & $(8862)$ \\
Average group & 0.0356 & 0.0231 & 0.0139 \\
Velocity & $(0.0346)$ & $(0.0226)$ & $(0.0135)$ \\
\hline \hline
\end{tabular}




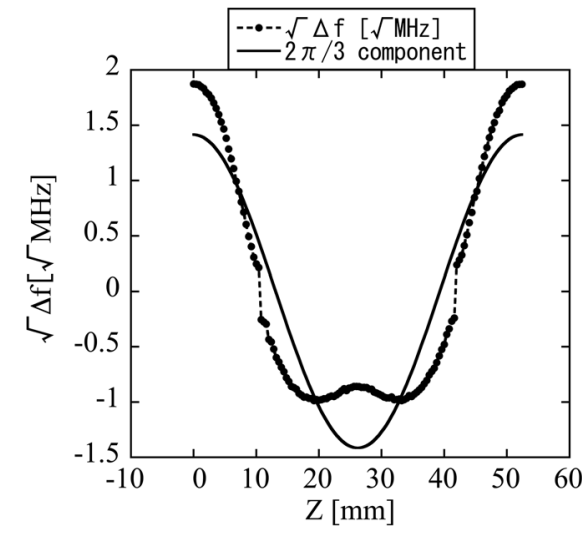

FIG. 6. Change in frequency, i.e., $\Delta f$ (acceleration-field pattern) as a function of the position of the bead and the TM01 $-2 \pi / 3$-mode acceleration-field pattern reconstructed from the $\Delta f$ data. The dashed line with the solid circles represents the on-axis acceleration electric-field pattern measured by the bead-pull perturbation method; the solid black curve is the reconstructed TM01 $-2 \pi / 3$ accelerating mode.

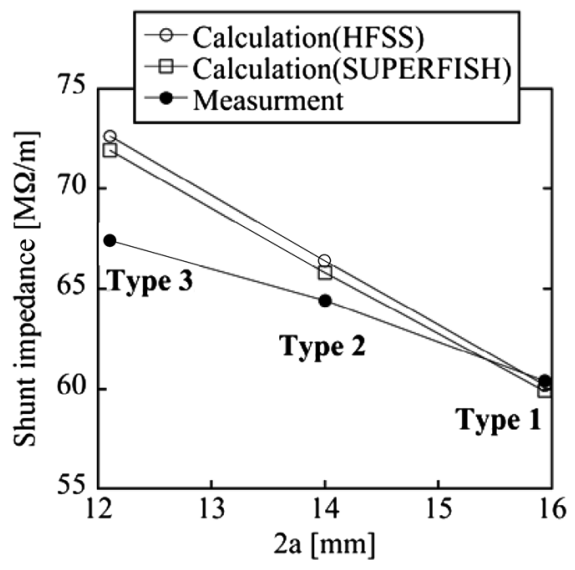

FIG. 7. Shunt impedances along the accelerating cells of the three test cavities. The solid circles denote the measurement results. The open circles and open squares denote the calculated results obtained by HFSS [24] and SUPERFISH [27], respectively.

$(\Delta f)$ as a function of the position of the bead moved along the center axis of the cavity. The $\Delta f$ curve corresponds to an electric-field pattern including the fundamental mode and its space harmonics. Fourier analysis reconstructs the acceleration-field pattern of the TM01 $-2 \pi / 3$ mode into the fundamental mode from the $\Delta f$ data. The shunt impedance, $R_{\mathrm{sh}}$, of the cavities derived from the $\Delta f$ data is expressed by

$$
R_{\mathrm{sh}}=\frac{|\Delta f| Q L}{4 \pi^{2} \epsilon a_{0}^{3} f_{0}^{2}},
$$

where $L$ is the length of the test cavity, $\varepsilon$ is the dielectric constant of the perturbing object, $a_{0}$ is the radius of the perturbing object $\left(\mathrm{a}_{0}=1 \mathrm{~mm}\right)$ and $f_{0}$ is the resonant frequency of the cavity [26]. Figure 7 shows the shunt impedance of the three types of test cavities. The solid circles in Fig. 7 denote the measurement results; the open circles and open squares denote the results of calculations using the simulation codes of HFSS [24] and SUPERFISH [27], respectively. In the measurement, the average shunt impedance of the cavities was $64 \mathrm{M} \Omega / \mathrm{m}$, which corresponds to $97 \%$ of the calculated value. The measured value of the shunt impedance is sufficient to attain the required high-acceleration gradient of the structure.

\section{FABRICATION AND PHASE ADJUSTMENT OF THE 1.8 M ACCELERATING STRUCTURE}

A 1.8-m-long accelerating structure was built on the basis of the above-mentioned experimental results obtained using the test cavities, as shown in Fig. 1. Copper blocks were used for the accelerating structure [class 1 oxygen-free copper treated with a hot isostatic pressing (HIP) process]. After all the disks and cylinder parts were machined, they were vertically stacked on a long V-block stage and brazed in a vacuum furnace. Then, a vacuum leak test and a hydraulic pressure resistance test were conducted to check for any vacuum leakage from the cell and the coupler as well as any water leakage from the cooling water channels.

To adjust the phase difference between the neighboring cells of the 1.8-m-long accelerating structure, the nodal shift method was used to measure the phase shift at each cell, and the dimpling method was adopted to tune the cell surface [20]. Finally, a nonresonant bead-pull perturbation method [28,29] was used to measure the electric field distribution and the cell frequency distributions along the cavity axis. Figure 8 shows

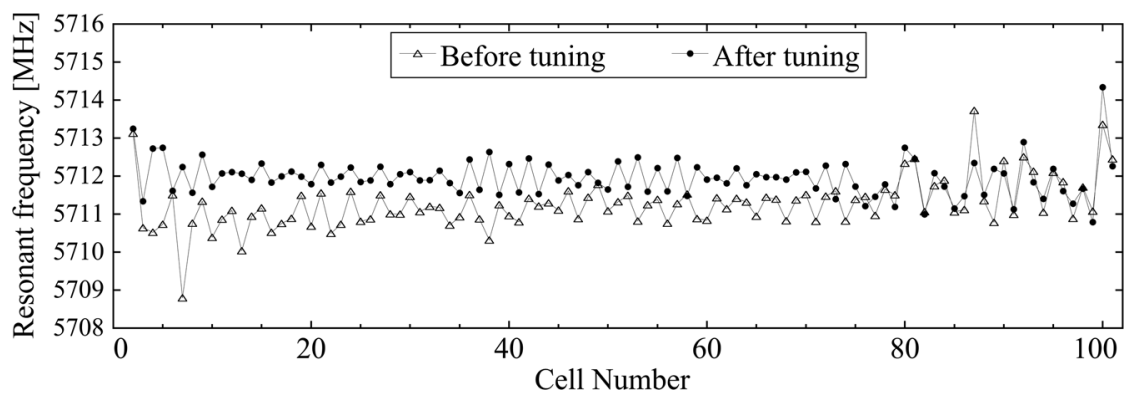

FIG. 8. Resonant frequency distribution along the individual accelerating cells before and after dimpling tuning. The curve with open triangles represents the result before tuning, and the curve with solid circles represents the result after tuning. 


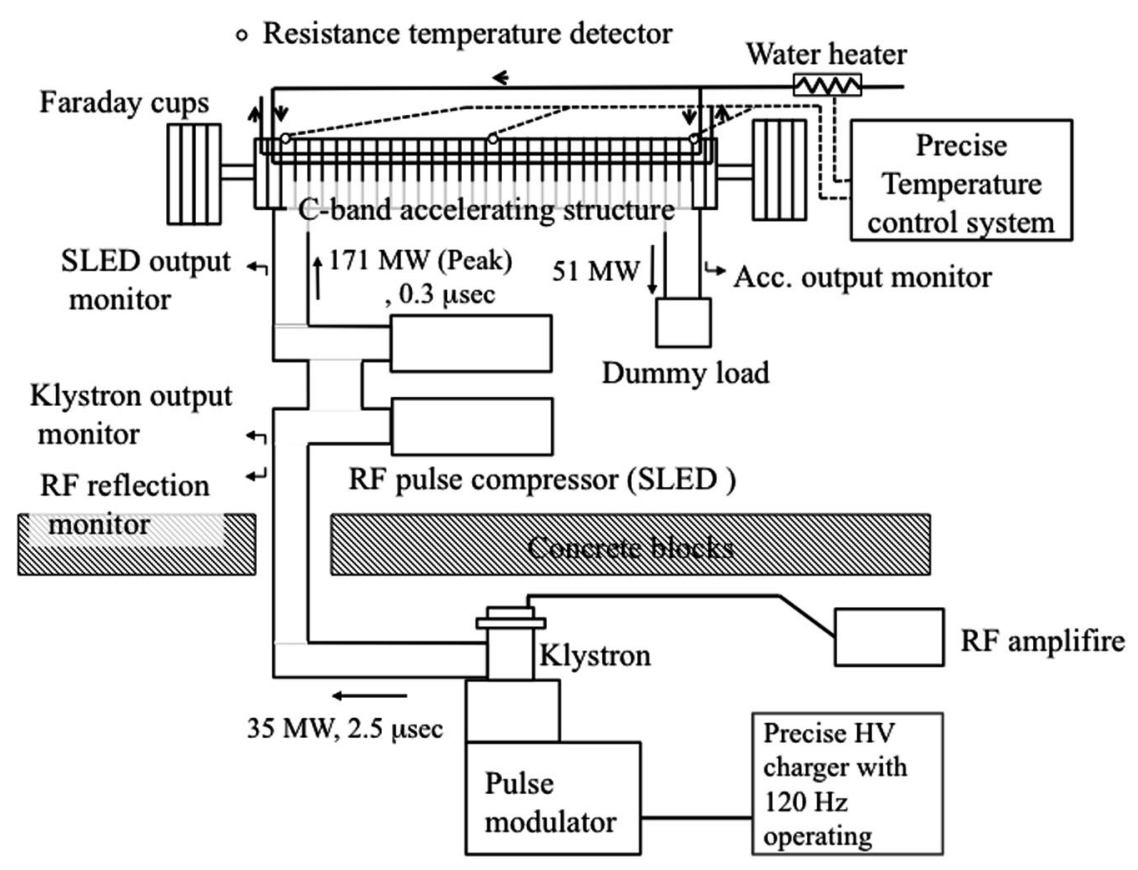

FIG. 9. Schematic diagram of the experimental setup for the high-power rf test of the accelerating structure.

the distributions of the individual cell frequencies of the structure before and after tuning by the dimpling method. Tuning using the nodal shift and bead-pull perturbation data was performed to adjust the cell frequencies to $5712 \mathrm{MHz}$ by pushing the cell walls through tuning holes. However, the resonant frequencies of some accelerating cells exceeded $5712 \mathrm{MHz}$ after brazing. Pulling the cell wall lowered these resonant frequencies. Finally, the tuning nearly adjusted the resonant frequency distribution along the accelerating structure to within $\pm 500 \mathrm{kHz}$ from $5712 \mathrm{MHz}$, as shown in Fig. 8 . The right side of Table I summarizes the performance achieved after tuning. We could obtain good results compared with the design parameters listed in Table I.

\section{HIGH-POWER RF TEST OF THE $C$-BAND STRUCTURE}

To evaluate the high-gradient performance of the structure, we performed a high-power rf test. We considered evaluating the following items in the high-power rf test.

1. The mitigation of the electric-field intensity on the surface of the ellipsoidal curved iris should be evaluated by measurements of the finally attained maximum acceleration gradient of the structure.

2. The dark current emitted from the inner surface of the structure should be measured to evaluate the inner surface roughness of the accelerating structure via measurement of the field enhancement factor $\beta$ [30].

3. Operation stability under the high-accelerationgradient condition should be checked by measuring the breakdown rate of the accelerating structure, which is related to 2 .
4. Our designed heat-dissipation value of the structure under the designed operation condition should be evaluated. Figure 9 shows a schematic diagram of the experimental setup of the high-power test for the accelerating structure. The configurations of the experimental instruments and the individual high-power rf components for the test, such as the waveguides and the rf-pulse compressor, namely the SLAC energy doubler (SLED) [31], are nearly the same as those of SACLA. The accelerating structure and SLED are installed in a test bunker surrounded by concrete blocks used as a radiation shield.

Figure 10 shows the rf waveforms under rf conditioning. The klystron output rf power and the rf-pulse width are $35 \mathrm{MW}$ and $2.5 \mu \mathrm{s}$, respectively. Because the SLED connected to the waveguide of the klystron multiplies its

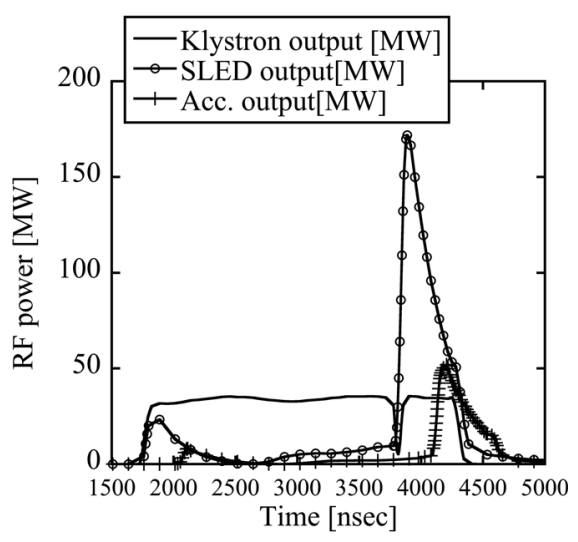

FIG. 10. Waveforms of the klystron output (solid line), the SLED output (open circle), and the output after passing through the accelerating structure (plus sign). 
output rf power by a factor of around 5, the peak power and the rf-pulse width of the SLED output become $171 \mathrm{MW}$ and $0.5 \mu \mathrm{s}$, respectively. The output rf pulse of SLED is fed into the input coupler of the accelerating structure. When the rf pulse passes though the accelerating structure, the peak rf power gradually decreases to $51 \mathrm{MW}$ at the output coupler owing to the wall loss of the structure. We calculated the acceleration gradient of the accelerating structure by Eq. (1), using the shunt impedance, $R_{\mathrm{sh}}$, and the attenuation constant, $\tau$. Our low-power rf measurements were obtained using these values. Here, the measured values of $R_{\mathrm{sh}}$ and $\tau$ are $64 \mathrm{M} \Omega / \mathrm{m}$ and 0.54 , respectively. The acceleration gradient calculated from the rf waveform data at the input coupler reaches $50.1 \mathrm{MV} / \mathrm{m}$.

To measure the amount of dark current emitted from the structure, we observed electron charges captured using two Faraday cups that were installed at the two ends of the accelerating structure. Figure 11 shows the amount of dark current as a function of the acceleration gradient. The dark current decreases as the rf conditioning time increases. After $385 \mathrm{~h}$, the amount of dark current becomes $150 \mathrm{pC} /$ pulse at $50 \mathrm{MV} / \mathrm{m}$. This dark current is small compared with that of the $C$-band choke-mode-type structure [32]. This result indirectly indicates that the dark current decreases proportionally with the intensity of the surface electric field. The field enhancement factor $(\beta)$, which is an index of the surface roughness under a high electric field, is a suitable parameter for evaluating the dark current emission. From the dark current data given in Fig. 11, we can calculate the field enhancement factor, $\beta$ by

$$
\frac{d\left(\log _{10} \frac{I}{E_{p}^{2.5}}\right)}{d\left(\frac{1}{E_{p}}\right)}=-\frac{2.84 \times 10^{9} \phi^{1.5}}{\beta},
$$

where $I$ is the amount of dark current, $E_{p}$ is the surface electric field strength, and $\phi$ is the work function of copper

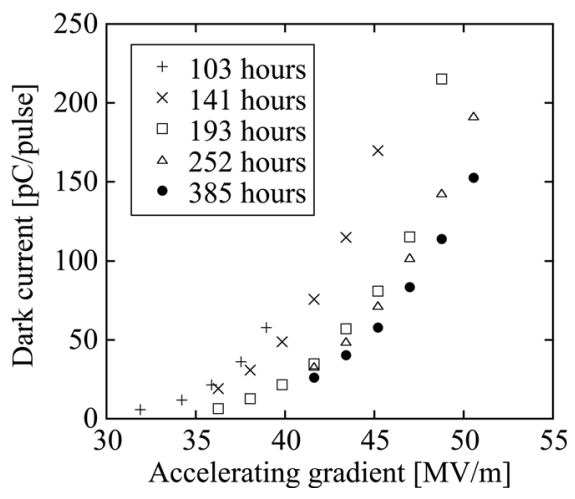

FIG. 11. Dark current emissions dependent on the acceleration gradients as the conditioning time changes. The plus signs, open crosses, open squares, open triangles, and solid circles corresponding to conditioning times of 103, 141, 193, 252 and $385 \mathrm{~h}$ after the start of conditioning, respectively.
$(4.5 \mathrm{eV})$ [30]. Finally, we obtained a $\beta$ value that was between 73 and 79 during rf conditioning of the accelerating structure. According to Refs. [30,32-35], the $\beta$ value is between 38 and 122 under a long operation time for $235-811 \mathrm{~h}$. Our obtained $\beta$ value is consistent with the values described in Refs. [30,32-35]. This fact indicates that the surface roughness of the ellipsoidal shape of the iris is sufficiently small for our purpose.

To measure the breakdown rate, the accelerating structure was continuously operated at the same acceleration gradient values for $24 \mathrm{~h}$. The measurement was performed at 256 and $370 \mathrm{~h}$ after the start time of the rf conditioning. Figure 12 shows the breakdown rate per day of the accelerating structure as a function of the acceleration gradient. This rate finally decreased to acceptable values for practical operation. We operated the structure at an acceleration gradient of $42 \mathrm{MV} / \mathrm{m}$; the breakdown rate with interlocked halts by electric discharges was once or less per day at $256 \mathrm{~h}$ after the conditioning started. Furthermore, the breakdown rate at $370 \mathrm{~h}$ and $50 \mathrm{MV} / \mathrm{m}$ decreased to around $40 \%$ of the breakdown rate at $256 \mathrm{~h}$. The achieved breakdown rate of the structure was once or less per day at around $42 \mathrm{MV} / \mathrm{m}$, which was the established acceleration gradient at SACLA. We can say that this rate is less than that of SACLA, because the new accelerating structure can operate at a higher acceleration gradient than SACLA while maintaining the same breakdown rate at SACLA. Furthermore, we compared the breakdown rate with those of the various accelerating structures described in Ref. [16] to analyze the breakdown behavior, as mentioned in the Introduction. We also checked whether the high-gradient characteristics of our developed structure are extensions of the characteristics of the ordinary accelerating structure. Figure 13 shows the breakdown rate per pulse as a function of the acceleration gradient. The unit of the breakdown rate is changed from count/day to bpp (breakdown per pulse). Here, the breakdown rate of the $C$-band structure at

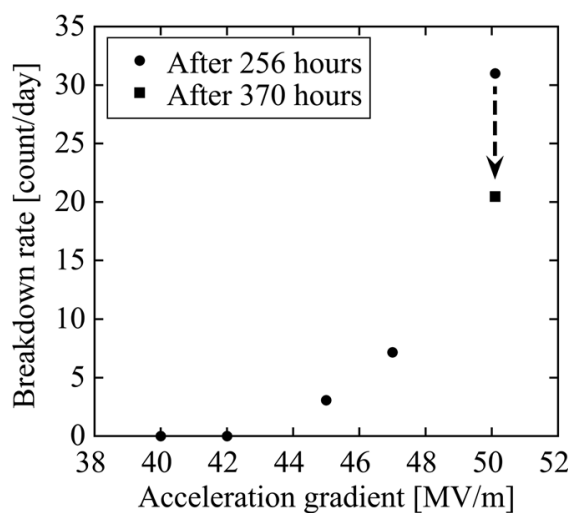

FIG. 12. Breakdown rate per day as a function of the acceleration gradient. The solid circles and solid squares denote the breakdown rates at 256 and $370 \mathrm{~h}$ after the start of conditioning, respectively. 


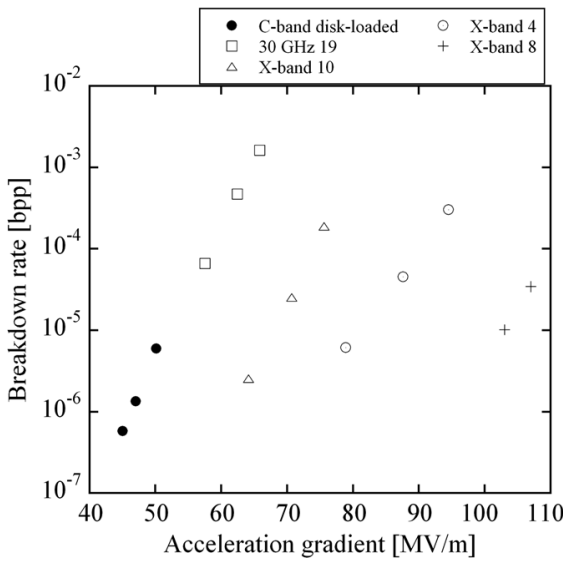

FIG. 13. Breakdown rate per pulse as a function of the acceleration gradient. The solid circles represent the case of the $C$-band disk-loaded-type accelerating structure, and the other markers represent the breakdown rates of the various structures in Ref. [16].

$50 \mathrm{MV} / \mathrm{m}$ is $6.0 \times 10^{-6} \mathrm{bpp}$. The relation between the acceleration gradient and the breakdown rate is given by

$$
E_{a}^{30} / B D R=\text { const }
$$

where $E_{a}$ is the acceleration gradient of the accelerating structure. The breakdown rates satisfy the condition of Eq. (7); thus, they are consistent with the results of the Ref. [16].

To investigate the thermal characteristics of the accelerating structure, we measured the heat dissipation value under 120 pps operation via calorimetric analysis using the cooling-water temperature difference between the inlet and outlet of the accelerating structure. During the measurement, the temperature of the accelerating structure was maintained within $30 \pm 0.01^{\circ} \mathrm{C}$ using a precise temperature control system. To measure the temperature of the accelerating structure, resistance temperature detectors are attached to the middle cell of the accelerating structure, as shown in Fig. 14(a). One of the measurement points (Point 1) is the bottom of a tuning hole (diameter, $1.5 \mathrm{~mm}$; depth, $10 \mathrm{~mm}$ ). Another point (Point 2) is the bottom of the tuning hole (diameter, $5 \mathrm{~mm}$; depth, $35 \mathrm{~mm}$ ). Figure 14(b) shows the temperature data at the points described in Fig. 14(a) and at the inlet and outlet of the cooling water when the acceleration gradient was $50 \mathrm{MV} / \mathrm{m}$. Here, the temperature difference between the inlet and outlet cooling water is $3.5 \mathrm{~K}$, and the heat dissipation value is $4.7 \mathrm{~kW}$ under an rf-pulse repetition rate of a 120 pps and an acceleration gradient of $50 \mathrm{MV} / \mathrm{m}$. This heat dissipation value is consistent with the value calculated from the difference between the input and output rf powers. In addition, when the temperature control system stabilized the temperature at Point 1 , the temperature at Point 2 was $0.5 \mathrm{~K}$ higher than that at Point 1, as shown in Fig. 14(b). This result is consistent with the model calculation of

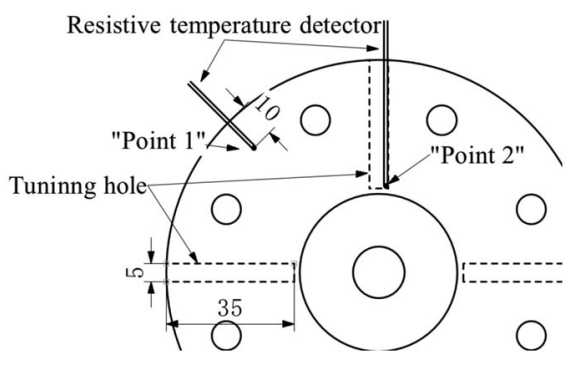

(a)

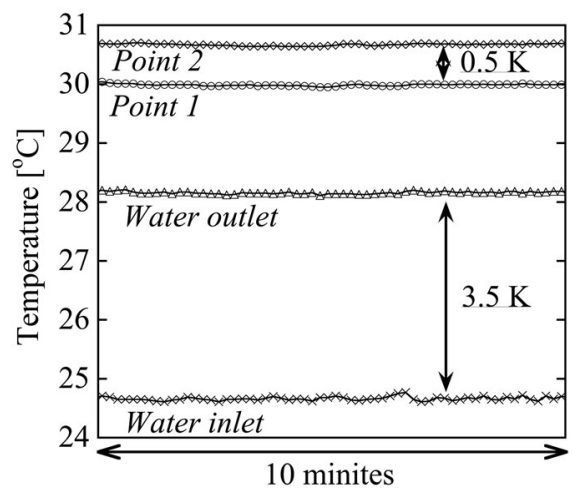

(b)

FIG. 14. (a) Schematic view of the measurement points where the resistive temperature detectors are located in the middle cell of the accelerating structure. (b) Temperature trends at "Point 1" and "Point 2" of the accelerating structure as well as the trends at the inlet and outlet of the cooling water when the acceleration gradient was $50 \mathrm{MV} / \mathrm{m}$.

the accelerating cell, as shown in Fig. 3. Furthermore, even if the rf-pulse repetition rate is changed, the phase change of the rf pulse that passes through the accelerating structure is $1^{\circ}$. The reduction in the acceleration gradient by this phase change is quite small (around $0.02 \%$ ) compared to our requirement. From the above-mentioned results, we believe that our cooling system design for the structure is optimum.

\section{ACCELERATION OF THE ELECTRON BEAM}

Ten columns of the $C$-band accelerating structures were installed as the main accelerator of an extreme ultraviolet (EUV)-FEL beam line [36]. To measure the acceleration gradient of the $C$-band accelerating structure, we measured the beam energy at the magnetic chicane consisting of four bending magnets, which is placed after the six accelerator columns. The experimental setup for this measurement is shown in Fig. 15(a). When we measured the beam energy, the rf phase of two 1.776-m-long columns (these accelerator columns are driven by one high-power klystron) was changed. Figure 15(b) shows the energy of an electron beam accelerated with the two columns corresponding to one unit, as a function of their rf phase. The beam was finally accelerated up to $147 \mathrm{MeV}$ with the crest phase. From the data, the acceleration gradient was calculated to 


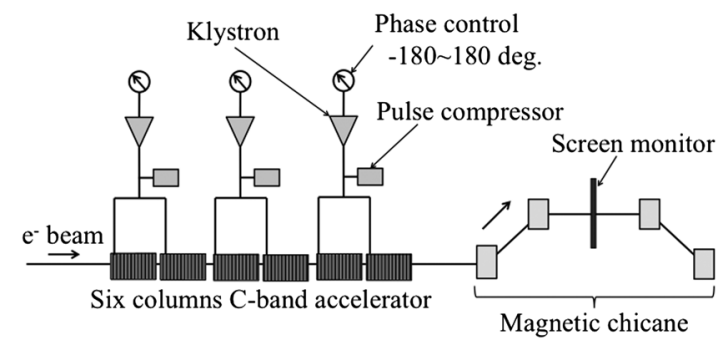

(a)

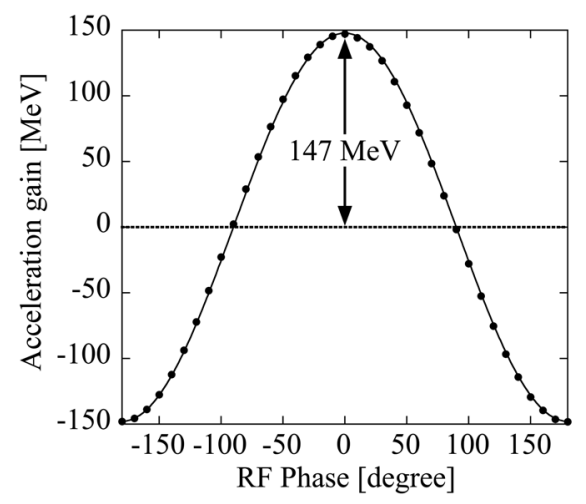

(b)

FIG. 15. (a) Schematic diagram of the $C$-band accelerator section in the EUV-FEL accelerator. (b) Acceleration energy gain of the two 1.8-m-long $C$-band accelerating structures as a function of the rf phase. The maximum acceleration energy gain is $147 \mathrm{MeV}$.

be $41.4 \mathrm{MV} / \mathrm{m}$. This acceleration gradient is consistent with the calculation using the measured rf power and the shunt impedance, as well as $\tau$ of the structure using Eq. (1). From the observation, we conclude that the required performance of the $C$-band accelerating structure has been realized.

\section{SUMMARY}

We developed a 1.8-m-long $C$-band disk-loaded-type accelerating structure that can be adopted with a high acceleration gradient of $>50 \mathrm{MV} / \mathrm{m}$ and operated with a high rf-pulse repetition rate of up to $120 \mathrm{pps}$. A cross section with an ellipsoidal curvature around an iris aperture was employed to reduce the strength of the electric field on the iris surface. After machining the structure, the actual dimensions of the iris ellipsoidal shape were compared with the ideal dimensions. The geometrical shape of the iris considerably overlapped with the ideal ellipsoidal shape within $10 \mu \mathrm{m}$. This agreement indicates a considerable possibility of reducing the electric field. Furthermore, the rf parameters of the manufactured $C$-band accelerating structure were checked. They were nearly consistent with our designed parameters. Next, the optimum arrangement of the water-cooling channels around the cell of the accelerating structure was determined according to the design for adapting to 120-pps operation. In this case, the maximum designed temperature on the cell surface was $35.2^{\circ} \mathrm{C}$ at an average heat load of $3.8 \mathrm{~kW}$, which was the designed heat dissipation value. A high-power rf test was performed to check the above-mentioned features of the accelerating structure. The results showed that the acceleration gradient of the structure reached $50.1 \mathrm{MV} / \mathrm{m}$. As evidence of the mitigation of the electric field strength of the iris surface, we measured the dark current at the downstream side of the accelerating structure. The dark current was sufficiently small compared with that of the $C$-band choke-mode-type structure. This means that the surface electric field of the developed structure is less than that of the $C$-band chokemode-type structure. The breakdown rate of the accelerating structure gradually decreased to acceptable values for our operation after a certain conditioning time. This rate was around once per hour at an acceleration gradient of $50 \mathrm{MV} / \mathrm{m}$ and an operational rf-pulse repetition rate of 120 pps. Furthermore, the breakdown rate of the $C$-band structure satisfied Eq. (7) and was consistent with the results presented in Ref. [16]. We confirmed that the highacceleration gradient characteristics of our developed accelerating structures are extensions of the characteristics of the ordinary structure. These results indicate that the surface electric field strength decreases because of the ellipsoidal curvature of the iris. Furthermore, the temperature difference between two points [Fig. 14(a)] in the middle cell of the accelerating structure was consistent with the result of a thermal calculation of the cell model. The average heat dissipation value was $4.7 \mathrm{~kW}$ at operating conditions of $120 \mathrm{pps}$ and $50 \mathrm{MV} / \mathrm{m}$. The measured heat dissipation value was different from the above-mentioned designed value because of the difference in the assumed input rf power. When considering the difference the measured heat dissipation value was identical to the designed value. It agreed well with the above-mentioned heat dissipation value calculated from the rf powers difference between the input and output parts. These results showed that the structure could correspond with 120-pps operation. This structure was finally installed as the main accelerator of our EUV-FEL accelerator. Then, in electron-beam acceleration, the acceleration gradient reached $41.4 \mathrm{MV} / \mathrm{m}$. The obtained acceleration gradient was in agreement with the result of the high-power rf test. From the above-mentioned facts, we can say that we succeeded in the development of a $C$-band diskloaded-type accelerating structure showing the required performance to achieve a high acceleration gradient of $>50 \mathrm{MV} / \mathrm{m}$ and an rf repetition rate of $120 \mathrm{pps}$. The $C$-band disk-loaded-type accelerating structure can be applied now to various electron accelerators involving reinforcement of the electron beam energy and reduced accelerator length.

\section{ACKNOWLEDGMENTS}

The authors wish to extend their gratitude to all staff members of SACLA and SPring-8 for their continuous support, especially Dr. T. Ishikawa and Dr. H. Tanaka for 
their management and promotion. The authors would also like to thank the SACLA operator group for maintenance and regular monitoring of the rf test stand.

[1] P. Emma et al., First lasing and operation of an angstromwavelength free-electron laser, Nat. Photonics 4, 641 (2010).

[2] T. Ishikawa et al., A compact X-ray free-electron laser emitting in the sub-angstrom region, Nat. Photonics 6, 540 (2012).

[3] M. Altarelli et al., DESY Report No. 2006-097, 2007.

[4] R. Ganter, SwissFEL Conceptual Design Report, Villigen, 2011, V19.

[5] J. H. Han, H. S. Kang, and I. S. Ko, Status of the PALXFEL Project, in Proceedings of the 3rd International Particle Accelerator Conference, New Orleans, LA, 2012 (IEEE, Piscataway, NJ, 2012).

[6] T. Bizen, T. Tanaka, Y. Asano, D. E. Kim, J. S. Bak, H. S. Lee, and H. Kitamura, Demagnetization of the undulator magnets irradiated high energy electrons, Nucl. Instrum. Methods Phys. Res., Sect. A 467-468, 185 (2001).

[7] R. B. Neal et al., The Stanford Two-miles Accelerator (W.A. Benjamin, Inc., New York, 1968).

[8] S. Reiche et al., Status of the SwissFEL facility at the Paul Scherrer Institute, in Proceedings of 33rd International Free Electron Laser Conference, Shanghai, China, 2011.

[9] T. Hara et al., Time-interleaved multienergy acceleration for an X-ray free-electron laser facility, Phys. Rev. ST Accel. Beams 16, 080701 (2013).

[10] T. Shintake et al., The choke mode cavity, Jpn. J. Appl. Phys. 31, L1567 (1992).

[11] T. Inagaki, C. Kondo, H. Maesaka, T. Ohshima, Y. Otake, T. Sakurai, K. Shirasawa, and T. Shintake, High gradient C-band linac for a compact X-ray free-electron laser facility, Phys. Rev. ST Accel. Beams 17, 080702 (2014).

[12] T. Kamitani et al., Status of C-band accelerator module in the KEKB injector LINAC, in Proceedings of the 22nd Particle Accelerator Conference, PAC-2007, Albuquerque, NM (IEEE, New York, 2007).

[13] D. Alesini et al., The C-band accelerating structure for SPARC photoinjector energy upgrade, J. Instrum. 8, P05004 (2013).

[14] R. Zennaro et al., Design, construction and power conditioning of the first C-band test accelerating structure for SwissFEL, in Proceedings of 26th Linear Accelerator Conference, Tel Aviv, Israel, 2012.

[15] W. Fang, D. Tong, Q. Gu, and Z. Zhao, Design and experimental study of a C-band travelling-wave accelerating structure, Chin. Sci. Bull. 56, 18 (2011).

[16] A. Grudiev, S. Calatroni, and W. Wuensch, New local field quantity describing the high gradient limit of accelerating structures, Phys. Rev. ST Accel. Beams 12, 102001 (2009).

[17] T. Taniuchi et al., Development of low dark current accelerating structure at SPring-8 Linac, in Proceedings of 6th Annual Meeting of Particle Accelerator Society in Japan, Tokai, Ibaragi, Japan (PASJ, Japan, 2009).

[18] V.A. Dolgashev et al., Report No. SLAC-PUB-10175, 2003.

[19] R. H. Miller et al., A damped detuned structure for the Next Linear Collider, in Proceedings of 18th Linear Accelerator Conference, Geneva, Switzerland, 1996, p. 644.

[20] W. J. Gallagher, SLAC-Project M Report No. M-205, 1960.

[21] V. F. Khan, A. D'Elia, R. M. Jones, A. Grudiev, W. Wuensch, G. Riddone, and V. Soldatov, Wakefield and surface electromagnetic field optimization of manifold damped accelerating structures for CLIC, Nucl. Instrum. Methods Phys. Res., Sect. A 657, 131 (2011).

[22] F. Wang, C. Adolphsen, and C. Nantista, Performance limiting effects in X-band accelerators, Phys. Rev. ST Accel. Beams 14, 010401 (2011).

[23] H. Tanaka et al., Bunch length variation and timing jitter caused by RF system instability in XFELSPring-8, in Proceedings of 4th Annual Meeting of Particle Accelerator Society in Japan, Wako, Saitama, Japan (PASJ, Japan, 2007).

[24] http://ansys.com.

[25] T. Hasegawa et al., Status of a precise temperatureregulation system for the C-band accelerator at XFEL/ SPring-8, in Proceedings of the International Particle Accelerator Conference, Kyoto, Japan (ICR, Kyoto, 2010).

[26] E. L. Ginzton, Microwave Measurements (McGraw-Hill Book Company, Inc., New York, 1957).

[27] http://laacg.lanl.gov/laacg.

[28] T. Khabiboulline et al., Internal Report No. DESY M-9502, 1995.

[29] H. Ego, H. Maesaka, T. Sakurai, Y. Otake, T. Hashirano, and S. Miura, Transverse C-band deflecting structure for longitudinal electron-bunch-diagnosis in XFEL "SACLA", Nucl. Instrum. Methods Phys. Res., Sect. A 795, 381 (2015).

[30] G. A. Loew et al., Report No. SLAC-PUB-4647, 1988.

[31] Z. D. Farkas et al., Report No. SLAC-PUB-1453, 1974.

[32] T. Sakurai et al., High power RF test on the C-band RF components of $8 \mathrm{GeV}$ accelerator for XFEL/SPring- 8 , in Proceedings of the 23rd Particle Accelerator Conference, Vancouver, Canada, 2009 (TRIUMF, Canada, 2009).

[33] Y. Igarashi et al., KEK Report No. 2003-9, 2004.

[34] T. Inagaki et al., High gradient test on the C-band chokemode type accelerating structure, in Proceedings of 1 st Annual Meeting of Particle Accelerator Society in Japan, Funahashi, Chiba, Japan (PASJ, Japan, 2004).

[35] T. Taniuchi et al., High power test of low dark current accelerating structure, High power test of low dark current accelerating structure, in Proceedings of the 8th Annual Meeting of Particle Accelerator Society of Japan, Tsukuba, Japan (PASJ, Japan, 2011).

[36] Y. Otake et al., Relocation and improvement status of the SCSS test accelerator to provide dual FEL drivers at SACLA, in Proceedings of 6th International Particle accelerator conference, Richmond, Virginia, USA, 2010. 